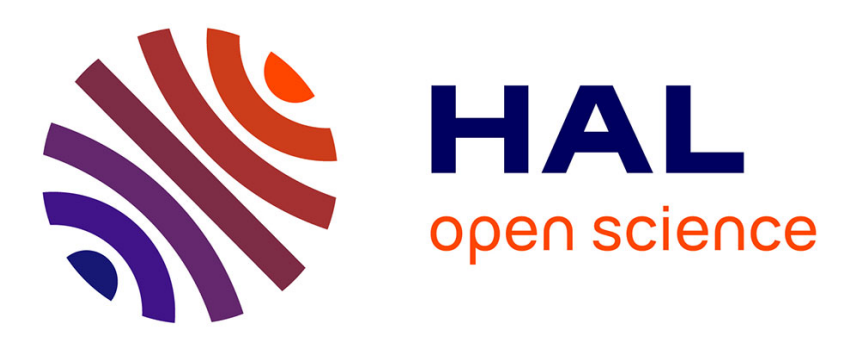

\title{
A sub-Riemannian modular approach for diffeomorphic deformations
}

\author{
Barbara Gris, Stanley Durrleman, Alain Trouvé
}

\section{To cite this version:}

Barbara Gris, Stanley Durrleman, Alain Trouvé. A sub-Riemannian modular approach for diffeomorphic deformations. 2nd conference on Geometric Science of Information, Oct 2015, Paris-Saclay, France. hal-01191540

\author{
HAL Id: hal-01191540 \\ https://hal.science/hal-01191540
}

Submitted on 2 Sep 2015

HAL is a multi-disciplinary open access archive for the deposit and dissemination of scientific research documents, whether they are published or not. The documents may come from teaching and research institutions in France or abroad, or from public or private research centers.
L'archive ouverte pluridisciplinaire HAL, est destinée au dépôt et à la diffusion de documents scientifiques de niveau recherche, publiés ou non, émanant des établissements d'enseignement et de recherche français ou étrangers, des laboratoires publics ou privés. 


\title{
A sub-Riemannian modular approach for diffeomorphic deformations
}

\author{
Barbara Gris ${ }^{1,2}$, Stanley Durrleman ${ }^{2}$, and Alain Trouvé ${ }^{1}$ \\ 1 CMLA, UMR 8536, École normale supérieure de Cachan, France \\ 2 Inria Paris-Rocquencourt, Sorbonne Universités, UPMC Univ Paris 06 UMR \\ S1127, Inserm U1127, CNRS UMR 7225, ICM, F-75013, Paris, France
}

\begin{abstract}
We develop a generic framework to build large deformations from a combination of base modules. These modules constitute a dynamical dictionary to describe transformations. The method, built on a coherent sub-Riemannian framework, defines a metric on modular deformations and characterises optimal deformations as geodesics for this metric. We will present a generic way to build local affine transformations as deformation modules, and display examples.
\end{abstract}

\section{Introduction}

A central aspect of Computational Anatomy is the comparison of different shapes, which are encoded as meshes or images. A common approach is the study of deformations matching one shape onto another, so that the differences between the two shapes are encoded by the deformation parameters $[9,11,17]$. In order to study differences between subjects on a particular structure, it should be useful to constrain locally the deformation, to favour realistic anatomic deformations, or to introduce some anatomical priors. For instance, for cortical surfaces with different sulci topography, one can prefer to favour lateral displacement over the creation of new sulci. Large deformations are commonly obtained through the integration of a vector field $[4,10,13,18]$ and a natural route is to introduce the constraints in the vector fields instead of the final diffeomorphism [19]. The vector field could be restricted, via a finite dimensional control variable, to a state dependent finite dimensional subspace generated by a finite basis and conceptualized in structures called hereafter deformation modules. Deformation modules should create interpretable deformations, and several modules should be allowed to combine to form more complex compound deformation modules in the spirit of Grenander's Pattern Theory [7].

Preliminary instantiations of the concept of deformation modules can be found in several early works. In the poly-affine framework $[3,14,20]$, deformations are created by the integration of a poly-affine stationary vector field. This vector field is a sum of few local affine transformations, which are then easily interpretable and share some features of deformation modules even if regions of each affine component are not updated during the deformation. In the LDDMM framework, a Riemannian structure is defined on the group of diffeomorphisms 
and optimal matchings are geodesics for this metric $[2,10]$. Several discretization schemes based on landmarks induce examples of finite dimensional state dependent representations of the velocity fields updated along the deformation and could be rephrased inside our definition of deformation modules. Note that the discretization scheme are thought of as approximations of the unconstrained nonparametrized infinite dimensional case. In a recently developed sparse-LDDMM framework $[6,12,15]$, the vector field is constrained to be a sum of a fixed number of local translations, carried by control points, with a more clearer focus on finite-dimensionality and local interpretability. Extension to locally more complex transformation are considered in $[8,16]$.

However, a consistent and general mathematical framework able to handle a large body of modular based large deformations is still missing in computational anatomy. A useful theory should not only provide a clear definition of deformation modules, but also explains how a hierarchy of deformation modules can be induced from basic one and how a Riemannian (or sub-Riemannian) setting can be defined underlying the computation of optimal large deformations and organizing the action of the different modules in the deformation process. This paper is a first attempt in that direction and presents a mathematical and computational sub-Riemannian framework to build large deformations from well defined deformation modules.

We will present several instances of deformation modules generating multiscale and locally affine vector fields as simple illustrative examples. We will show trajectories that can be built from the combination of such modules, and how the component of a particular module can be recovered and followed through the integration.

\section{Definition of a deformation module}

Intuitively, a deformation module creates a vector field parametrized in low dimension, describing a distinctive aspect of a larger deformation pattern. This notion should embrace at least the notion a sum of local translations, scalings or other local affine transformations as simple examples. In the following, $C_{0}^{1}\left(\mathbb{R}^{d}\right)$ will be the set on $C^{1}$ continuous mapping $v$ vanishing at infinity equipped with the usual supremum norm on $v$ and its first derivative, and Diff ${ }_{0}^{1}\left(\mathbb{R}^{d}\right)$ the open subset of Id $+C_{0}^{1}\left(\mathbb{R}^{d}\right)$ of $C^{1}$ diffeomorphisms. We recall that for any curve $v \in L^{1} \doteq L^{1}\left([0,1], C_{0}^{1}\left(\mathbb{R}^{d}\right)\right)$ there exists a unique curve $t \mapsto \phi_{t}^{v} \in \operatorname{Diff}_{0}^{1}\left(\mathbb{R}^{d}\right)$ solution of the flow equation $\dot{\phi}_{t}^{v}=v_{t} \circ \phi_{t}^{v}$, with $\phi_{0}^{v}=\mathrm{id}$.

Let $O$ be a finite dimensional manifold and $(\phi, o) \mapsto \phi . o$ a $C^{1}$ action of $\operatorname{Diff}_{0}^{1}\left(\mathbb{R}^{d}\right)$ on $O$ in the sense that $(\phi, o) \mapsto \phi . o$ is continuous and there exists a continuous mapping $\xi: O \times C_{0}^{1}\left(\mathbb{R}^{d}\right) \rightarrow T O$ called the infinitesimal action, so that $v \mapsto \xi_{o}(v) \doteq \xi(o, v)$ is linear continous, $o \mapsto \xi_{o}$ is locally Lipschitz and for any $v \in L^{1}$, the curve $t \mapsto o_{t} \doteq \phi_{t}^{v}$. oo is absolutly continuous (a.c.) and satisfies $\dot{o}_{t}=\xi_{o_{t}}\left(v_{t}\right)$ for almost every $t \in[0,1]$.

Remark 1. In fact $O$ is a shape space as defined by S. Arguillère in [2]. 
Definition 1 (Deformation module). We say that $M=(O, H, V, \zeta, \xi, c)$ is a deformation module with geometrical descriptors in $O$, controls in $H$, infinitesimal action $\xi$, field generator $\zeta$ and cost $c$ if $H$ is a finite dimensional Euclidean space, $V$ is an Hilbert space with $V \stackrel{C^{0}}{\hookrightarrow} C_{0}^{1}\left(\mathbb{R}^{d}\right), \zeta: O \times H \rightarrow O \times V$ is a continuous mapping such that $h \mapsto \zeta_{o}(h)$ is linear where $\zeta(o, v)=\left(o, \zeta_{o}(v)\right), o \mapsto \zeta_{o}$ is locally Lipschitz and $c: O \times H \rightarrow \mathbb{R}^{+}$is a continuous mapping such that $h \mapsto c_{o}(h) \doteq c(o, h)$ is a positive quadratic form on $H$ and there exists $C>0$ such that for each $o, h$ :

$$
\left|\zeta_{o}(h)\right|_{V}^{2} \leq C c_{o}(h)
$$

Let us explain how a deformation modules induces large deformations.

Definition 2 (Finite energy controled paths on $O$ ). Let $a, b \in O$. We denote $\Omega_{a, b}$ the set of mesurable curves $t \mapsto\left(o_{t}, h_{t}\right) \in O \times H$ where $o_{t}$ is a.c., starting from $a$ and ending at $b$, such that $\dot{o}_{t}=\xi_{o_{t}}\left(v_{t}\right)$ for $v_{t} \doteq \zeta_{o_{t}}\left(h_{t}\right)$ and $E(o, h) \doteq$ $\int_{0}^{1} c_{o_{t}}\left(h_{t}\right) d t<\infty$ where $E(o, h)$ is called the energy of $(o, h)$.

Thanks to (1) and the smoothness condition for deformation modules one get the following construction of flows :

Proposition 1 (Flows generated by a deformation module). Let $(o, h) \in \Omega_{a, b}$ and $v=\zeta_{o}(h)$. Then $\int_{0}^{1}\left|v_{t}\right|_{V}^{2} d t \leq C \int_{0}^{1} c_{o_{t}}\left(h_{t}\right) d t<\infty$ so that $v \in L^{2}([0,1], V) \subset$ $L^{1}$ and $o_{t}=\phi_{t}^{v} . o_{0}$. Moreover, $\int_{0}^{1}\left|h_{t}\right|_{H}^{2} d t \leq\left(\sup _{t}\left\|c_{o_{t}}^{-1}\right\|\right) \int_{0}^{1} c_{o_{t}}\left(h_{t}\right) d t<\infty$ (with $\left.\left\|c_{o}^{-1}\right\| \doteq \sup _{|h|_{H}=1} c_{o}(h)^{-1}\right)$ so that $h \in L^{2}([0,1], H)$.

A more geometrical point of view on deformation modules would be to identify $\zeta$ (resp. $\xi$ ) as a continuous morphisms between the two vector bundles $O \times H$ and $O \times V$ (resp. $O \times V \rightarrow T O)$ and $c$ as a metric on $O \times H$. Now, $\rho \doteq \xi \circ \zeta: O \times H \rightarrow T O$ and $c$ induce a sub-Riemannian structure on $O$ (as defined in [1]). Moreover, indexed by the choice of $a \in O$, we can induce a sub-Riemannian structure on $\operatorname{Diff}_{0}^{1}\left(\mathbb{R}^{d}\right)$ by considering $\rho^{a}: \operatorname{Diff}_{0}^{1}\left(\mathbb{R}^{d}\right) \times H \rightarrow$ $T \operatorname{Diff}_{0}^{1}\left(\mathbb{R}^{d}\right)=C_{0}^{1}\left(\mathbb{R}^{d}\right)$ such that $\rho_{\phi}^{a}(h) \doteq \zeta_{\phi . a}(h)$ and the metric on $\operatorname{Diff}_{0}^{1}\left(\mathbb{R}^{d}\right) \times H$ given by $c_{\phi}^{a}(h) \doteq c_{\phi . a}(h)$.

During the trajectory, the geometrical descriptor $o_{t}$ creates the vector field $\zeta_{o}(h)$ which acts back on $o_{t}$ through the infinitesimal action $\xi$. Then, as explained in figure $1, \xi$ can be seen as a feedback action, allowing geometrical descriptors to evolve with the vector field.

\subsection{First example : sum of local translations}

This first example explains how the construction of [6] can be seen as a deformation module. Let $\sigma \in \mathbb{R}^{+}$, and $D \in \mathbb{N}$, we want to build a module $M$ that would generate a sum of $D$ local translations acting at scale $\sigma$. We set $O=\left(\mathbb{R}^{d}\right)^{D}$ (families of $D$ points), $H=\left(\mathbb{R}^{d}\right)^{D}$ (families of $D$ vectors) and $V=V_{\sigma}$ the scalar Gaussian RKHS of scale $\sigma$. For $o=\left(z_{i}\right) \in O$, we define $\zeta_{o}: h=\left(\alpha_{i}\right) \in H \mapsto \sum_{i=1}^{D} K_{\sigma}\left(z_{i}, \cdot\right) \alpha_{i}, \xi_{o}: v \in V \mapsto\left(v\left(z_{i}\right)\right)_{i}$ (application of the vector field at each point) and $c_{o}: h=\left(\alpha_{i}\right) \in H \mapsto\left|\sum_{i} K_{\sigma}\left(z_{i}, \cdot\right) \alpha_{i}\right|_{V_{\sigma}}^{2}$. 


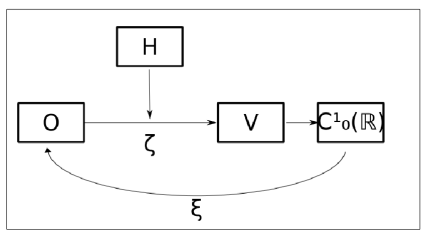

Fig. 1. Schematic view of a deformation module.

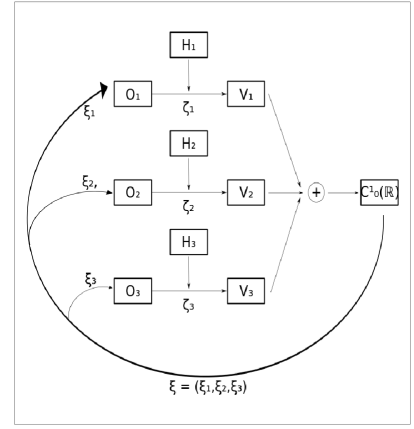

Fig. 2. Schematic view of a combination of deformation modules.

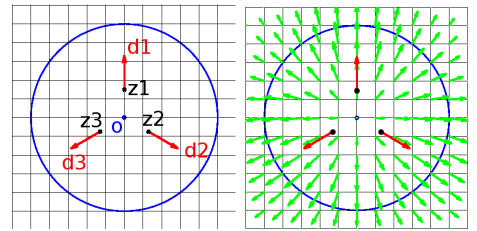

Fig. 3. Local scaling. Left : Geometrical descriptor $o$ (in blue) and intermediate tools (in black and red). Right: Plot of the resulting vector field in green.

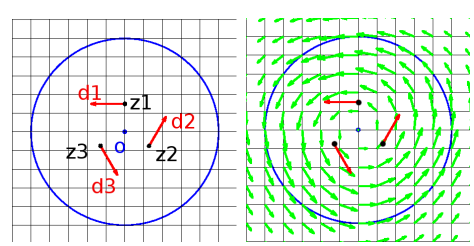

Fig. 4. Local rotation. Left : Geometrical descriptor $o$ (in blue) and intermediate tools (in black and red). Right: Plot of the resulting vector field in green.

\subsection{Second example : constrained local affine transformations}

We present here a generic way to build a particular kind of local affine transformation as a deformation module. Let us first start by an illustrative example of a local scaling in dimension 2 parametrized by a scale $\sigma$, a center $o$ and a scaling ratio $h$. From $\sigma$ and $o$, we build 3 points $z_{j}$ and 3 unit vectors $d_{j}$ as described in Figure 3. We can then define the vector field generated by the geometrical descriptor $o$ and the control $h$ by $\zeta_{o}(h) \doteq \sum_{j=1}^{3} K_{\sigma}\left(z_{j}(o, \sigma), \cdot\right) d_{j}(o, \sigma) h$. We emphasize here that points $z_{j}$ and vectors $d_{j}$ are intermediate tools used to build the vector field but that the latter is only parametrized by $\sigma, o$ and $h$. We can then define the module $M$ by the following spaces $: V=V_{\sigma}, O=\mathbb{R}^{d}$, $H=\mathbb{R}$ and applications, for $o \in O: \zeta_{o}, \xi_{o}: v \in C_{0}^{1}\left(\mathbb{R}^{d}\right) \mapsto \frac{1}{3} \sum_{j=1}^{3} v\left(z_{j}(o, \sigma)\right)$ and $c_{o}: h \in H \mapsto\left|\zeta_{o}(h)\right|_{V_{\sigma}}^{2}=\sum_{j, j^{\prime}} K_{\sigma}\left(z_{j}, z_{j^{\prime}}\right) d_{j}^{T} d_{j^{\prime}} h^{2}$.

If we change the rule to build vectors $d_{j}$, we can build a local rotation, see Figure 4. More generally, we can set any kind of rules to build points $z_{j}$ and unit vectors $d_{j}$ from a geometrical descriptor $o$ to create another type of local transformation. We have here defined a generic way to build a module that generates a vector field based on user's assumptions. It is the way to incorporate anatomical prior in the deformation. 


\subsection{Third example : unconstrained local affine transformations}

Some more complex local affine transformations can be needed. Any local affine deformation in dimension $d$ can be approximated by a sum of $d+1$ local translations carried by points close to each other with respect to the scale. In this spirit we can build another type of module, defining local affine transformations in $P$ different areas of size defined by the same scale $\sigma$, by summing $D \doteq P \times(d+1)$ local translations, whose centres would be assembled in groups of $d+1$ points. We can build a module corresponding to the sum of $D$ local translations, as in section 2.1. This example differs from [6] as we suppose that $(d+1)$ centres of translations are pooled together. This construction allows to build modules that generate a vector field which is locally an affine transformation, without prior constraints. This module class differs from the poly-affine framework in that the neighbourhood which is affected by the local module is transported by the global transformation (via $\xi$ ).

\section{Combination of modules}

We want to define the combination of $L$ modules so that it remains a module in the sense of Definition 1. Modules are defined by spaces $O^{l}, H^{l}, V^{l}$, and applications $\zeta^{l}, \xi^{l}$ and $c^{l}$ for each $l=1 \cdots L$. We can define $\pi: w=\left(w_{1}, \ldots, w_{L}\right) \in$ $W \doteq \prod_{l} V^{l} \mapsto \sum_{i} w_{i} \in C_{0}^{1}\left(\mathbb{R}^{d}\right)$. One can show that $V \doteq \pi(W)$ is a Hilbert space and is continuously embedded in $C_{0}^{1}\left(\mathbb{R}^{d}\right)$, with for $v \in V,|v|_{V}^{2}=\inf \left\{\sum_{i}\left|v_{i}\right|_{V^{i}}^{2} \mid\right.$ $\left.\pi\left(\left(v_{i}\right)_{i}\right)=v\right\}$. We are then able to define the compound module $M$ by spaces : $O \doteq \prod_{l} O^{l}, H \doteq \prod_{l} H^{l}, V=\operatorname{Im}(\pi)$ and applications, for $o=\left(o^{l}\right)_{l} \in O: \zeta_{o}: h=$ $\left(h^{l}\right) \in H \mapsto \pi\left(\left(\zeta_{o^{l}}^{l}\left(h^{l}\right)\right)_{l}\right)=\sum_{l} \zeta_{o^{l}}^{l}\left(h^{l}\right) \in V, \xi_{o}: v \in C_{0}^{1}\left(\mathbb{R}^{d}\right) \mapsto\left(\xi^{l}\left(o^{l}, v\right)\right)_{l} \in T_{o} O$ and $c_{o}: h=\left(h^{l}\right) \in H \mapsto \sum_{l} c_{o^{l}}^{l}\left(h^{l}\right)$. Then for any $h=\left(h^{l}\right) \in H$ we have

$$
\left|\zeta_{o}(h)\right|_{V}^{2} \leq \sum_{l=1}^{L}\left|\zeta_{o^{l}}^{l}\left(h^{l}\right)\right|_{V_{l}} \leq \sum_{l=1}^{L} C_{l} c_{o^{l}}^{l}\left(h^{l}\right) \leq\left(\max _{l \in L} C_{l}\right) c_{o}(h) .
$$

All necessary hypotheses to build a module are satisfied. A schematic view of this combination can be seen on figure 2 . Note that even if the cost of the elementary module for each $l$ is given by $c_{o^{l}}^{l}\left(h^{l}\right)=\left|\zeta_{o^{l}}^{l}\left(h^{l}\right)\right|_{V^{l}}^{2}$, as in our following examples, the cost of the compound module is then $c_{o}(h)=\sum_{l}\left|\zeta_{o^{l}}^{l}\left(h^{l}\right)\right|_{V^{l}}^{2} \neq|\zeta(h)|_{V}^{2}$ so that that generically (when $\pi$ is not one to one) $C>1$ and $c$ is not the pull-back metric on $O \times H$ of the metric on $O \times V$. In alternative extensions of sparseLDDMM to locally more complex transformation ([8],[16]) the norm of $V$ has been a natural choice for the cost. Then the cost depends on the built vector field but not on the way it is built, unlike in our construction. Here minimizing the cost corresponds to selecting one way of building the vector field, and then choosing a particular cost enables to favour certain decomposition over others.

\subsection{An example of combination: sum of multi-scale translations}

Let us build a module $M$ which would be a sum of local translations, acting at different scales $\sigma_{l}$. For each $\sigma_{l}$ can be built a module of type defined in 2.1, let 
$D_{l} \in \mathbb{N}$ be the number of local translations acting at this scale. The multi-scale module is then the combination of these modules. In particular the cost is, for $o=\left(z_{j}^{l}\right)$ and $h=\left(\alpha_{j}^{l}\right), c_{o}(h)=\sum_{l} \sum_{j} K_{\sigma_{l}}\left(z_{j}^{l}, z_{j^{\prime}}^{l}\right) \alpha_{j}^{l T} \alpha_{j^{\prime}}^{l}$. It is clear here that it is not derived from the norm of the vector field $\zeta_{o}(h)=\sum_{l=1}^{N} \sum_{j=1}^{D_{l}} K_{\sigma_{l}}\left(z_{j}^{l}, \cdot\right) \alpha_{j}^{l}$ in $V$, which is here the RKHS of kernel $\sum_{l} K_{\sigma_{l}}$, as in [12] but from the way it is built as sum of elements of $V_{o^{l}}^{l}=\zeta_{o^{l}}^{l}\left(H^{l}\right)$.

\section{Shooting}

Let us consider a generic deformation module $M$ and fix two values $a, b \in O$. For each trajectory $(o, h) \in \Omega_{a, b}$ (see Def. 2) we get a flow $\phi^{v}$ with $v=\zeta_{o}(h)$ and $\int_{0}^{1}\left|v_{t}\right|_{V} d t \leq C E(o, h)<\infty$ (see Prop. 1).

We assume that for a distance $d_{O}$ compatible with the topology on $O$, there exists $\gamma>0$ and $K \subset \mathbb{R}^{d}$ such that $d_{O}\left(\phi . a, \phi^{\prime} . a\right) \leq \gamma\left\|\phi-\phi^{\prime}\right\|_{\infty, K}$ where \|\|$_{\infty, K}$ is the uniform $C^{1}$ norm on $K$. Note that this property is verified in our examples.

Theorem 1. If $\Omega_{a, b}$ is not empty, then $E$ reaches its minimum on $\Omega_{a, b}$.

Proof. (Sketch) One consider a minimizing sequence $\left(o^{n}, h^{n}\right) \in \Omega_{a, b}$ and the associated flows $\phi^{v_{n}}$ for $v_{n} \doteq \zeta_{o^{n}}\left(h^{n}\right)$. Since $\int_{0}^{1}\left|v_{t}^{n}\right|_{V}^{2} \leq C E\left(o^{n}, h^{n}\right)$ we can assume (up to the extraction of a subsequence) that $v^{n}$ weakly converges to $v^{\infty}$ so that $t \mapsto \phi_{t}^{v^{n}}$ converges uniformly for the \|\|$_{\infty, K}$ norm and $o^{n}$ converges uniformly to $o^{\infty}$. Thus there exists a compact $K^{\prime} \subset O$ such that $o^{\infty}$ and the $o^{n}$ stay in $K^{\prime}$. Now, we have that $\int_{0}^{1}\left|h_{t}^{n}\right|_{H}^{2} d t \leq \sup _{K^{\prime}}\left\|c_{o}^{-1}\right\| E\left(o^{n}, h^{n}\right)$ so that (up to the the extraction of a subsequence) we can assume that $h^{n}$ weakly converges to $h^{\infty}$. Hence, for any $w \in L^{2}([0,1], V)$ we have $\left|\int_{0}^{1}\left\langle v_{t}^{\infty}-\zeta_{o_{t}^{\infty}}\left(h_{t}^{\infty}\right), w_{t}\right\rangle_{V} d t\right| \leq$ $\varlimsup \overline{\lim }\left|\int_{0}^{1}\left\langle\left(\zeta_{o_{t}^{\infty}}-\zeta_{o_{t}^{n}}\right)\left(h_{t}^{n}\right), w_{t}\right\rangle_{V} d t\right| \leq \overline{\lim }\left(\int_{0}^{1}\left|w_{t}\right|_{V}^{2} d t \int_{0}^{1}\left\|\zeta_{o_{t}^{\infty}}-\zeta_{o_{t}^{n}}\right\|^{2}\left|h_{t}^{n}\right|_{H}^{2} d t\right)^{1 / 2}=$ 0 . Since $w$ is arbitrary, $v^{\infty}=\zeta_{o_{t}^{\infty}}\left(h_{t}^{\infty}\right)$ and $\dot{o}_{t}^{\infty}=\xi_{o_{t}}\left(v_{t}\right)$ so that $\left(o^{\infty}, h^{\infty}\right) \in \Omega_{a, b}$. Now, $\int_{0}^{1} c_{o_{t}^{\infty}}\left(h_{t}^{\infty}\right) d t \leq \underline{\lim }\left(\int_{0}^{1} c_{o_{t}^{\infty}}\left(h_{t}^{n}\right) d t \int_{0}^{1} c_{o_{t}^{\infty}}\left(h_{t}^{\infty}\right) d t\right)^{1 / 2}$ where we have used that $c_{o}(h)$ can be written as $\left\langle C_{o} h, h\right\rangle_{H}$ where $o \mapsto C_{o}$ is continous and that $h^{n}$ is weakly converging in $L^{2}([0,1], H)$. Since $\left|\int c_{o_{t}^{\infty}}\left(h_{t}^{n}\right)-c_{o_{t}^{n}}\left(h_{t}^{n}\right) d t\right| \leq\left(\sup _{t} \| C_{o_{t}^{\infty}}-\right.$ $\left.C_{o_{t}^{n}} \|\right) \int_{0}^{1}\left|h_{t}^{n}\right|_{H} d t \rightarrow 0$ we get $\int_{0}^{1} c_{o_{t}^{\infty}}\left(h_{t}^{\infty}\right) d t \leq \underline{\lim } \int_{0}^{1} c_{o_{t}^{n}}\left(h_{t}^{n}\right) d t$.

A trajectory of $\Omega_{a, b}$ minimizing $E$ can be obtained from the Hamiltonian and optimal control point of view [2] that we briefly describe below. Let us define the dual variable $\eta \in\left(T_{o} O\right)^{*}$ and introduce the Hamiltonian $H(\eta, o, h)=$ $\langle\eta, \xi \circ \zeta(o, h))\rangle-\frac{1}{2} c_{o}(h)$. It can be shown that trajectories of $\Omega_{a, b}$ minimizing $E$ can be separated in two categories : normal and abnormal geodesics, we will concentrate on normal geodesics as justified at the end of this section. Normal geodesics are such that there exists a trajectory $(o, \eta)$ in $T^{*} O$ such that (in a local chart)

$$
\left\{\begin{array}{l}
\dot{o}=\xi\left(o, \zeta_{o}(h)\right) \\
\dot{\eta}=-\frac{\partial H}{\partial o}(\eta, o, h) \\
h=A(o) \eta
\end{array}\right.
$$



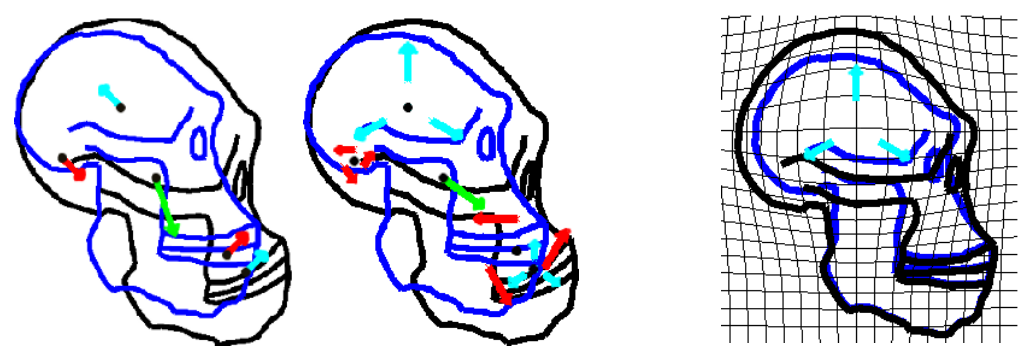

Fig. 5. Initial positions for modules : in red rotations, Fig. 6. In blue the initial in green translation and in cyan scaling. In blue is shape, in cyan are intermethe initial shape, in black the shape at $t=1$. Left: diate tools useful to build parametrization of the vector field : initial geometrical the vector field at $t=0$, descriptors (in black) and momenta. Right: Initial ge- in black is the transported ometrical descriptors, controls and intermediate tools. shape while following the

largest scaling.

where $A(o)$ is a matrix depending on $o$. The whole geodesic trajectory is then parametrized by initial values of $(o, \eta)$ (of dimension twice the dimension of $o$ ). We have obtained a geodesic shooting from an element $\left(a, \eta_{0}\right)$, with $\eta_{0} \in\left(T_{a} O\right)^{*}$, to a geodesic path $(o, h)$ and then to a trajectory $\phi^{v}$ with $v=\zeta_{o}(h)$.

Remark 2. In practice we do not minimize $E$ with fixed a end-point but minimize $J\left(o_{t=0}, h\right)=E(o, h)+g\left(o_{t=1}\right)$ (with o such that $\dot{o}=\xi_{o}\left(\zeta_{o}(h)\right)$ ). Trajectories minimizing $J$ are normal geodesics following equations 2 (see [2]).

\section{$5 \quad$ Examples}

\subsection{Shooting with constrained types of local affine transformations}

We present here an example of geodesic trajectory created by the combination of different modules (in dimension 2): one translation (scale 100), two rotations (scales 20 and 60), two scaling (scales 50 and 20). Initial values of the geometrical descriptor (5 points : dimension 10) and the momentum (same dimension as $o$ ) define the total trajectory: it is parametrized in dimension 20. These parameters are displayed in Figure 5 as well as initial controls (lengths of directions $d_{j}$ for scaling and rotation), intermediate tools $\left(z_{j}, d_{j}\right)$ and the transported shape. We can follow the action of a particular module $M_{l}$ by fixing the trajectory of its controls $h^{l}$, and integrating the new trajectory $\left(\tilde{v}, \tilde{o}^{l}\right)$ such that: $\tilde{o}^{l}(t=0)=$ $o^{l}(t=0)$ and for each $t: \tilde{v}(t)=\zeta_{\tilde{o}^{l}(t)}^{l}(h(t)), \dot{\tilde{o}}^{l}(t)=\xi_{\tilde{o}^{l}(t)}^{l}(\tilde{v}(t))$.

\subsection{Matching with unconstrained local affine transformations}

We present here the matching from a shape $f_{0}$ to another $f_{1}$, using a compound module of unconstrained local affine transformations. We set $\sigma_{1}=60, \sigma_{2}=20$, 

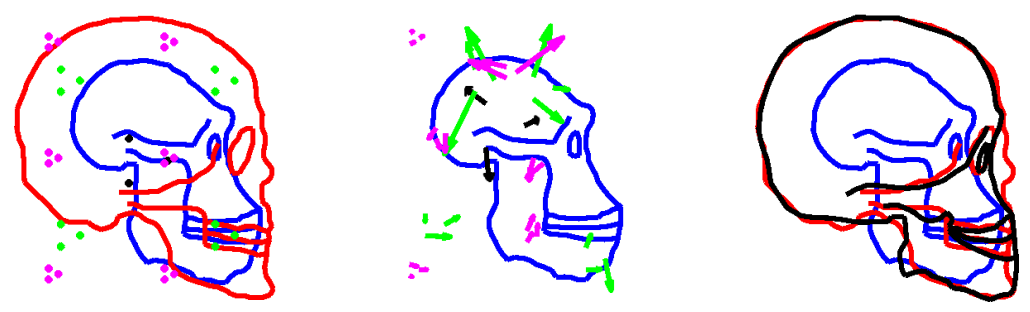

Fig. 7. Matching from $f_{0}$ (blue) to $f_{1}$ (red), with parameters per scale $\left(\sigma_{1}\right.$ : black, $\sigma_{2}$ :green, $\sigma_{3}:$ magenta). Left: initialization geometrical descriptors. Middle: Optimized initial geometrical descriptors and controls. Right : Match at $t=1$ (black).

$\sigma_{3}=8$ three scales and $P_{1}=1, P_{2}=9, P_{3}=20$ the number of groups of local translations at each scale. We define $O, H, V, \zeta, \xi$ and $c$ as in section 3.1. To each $(a, \eta) \in T O^{*}$, can be associated a geodesic trajectory of controls $h^{a, \eta}$ and then a diffeomorphism $\phi^{a, \eta}$ as defined in 4 . The matching problem corresponds then to finding values of $a$ and $\eta$ minimizing $E\left(a, \eta, f_{0}, f_{1}\right)=c_{a}\left(h_{0}^{a, \eta}\right)+\lambda D\left(\phi_{1}^{a, \eta} \cdot f_{0}, f_{1}\right)$ where $D$ is the varifold distance [5]. Our first implementation is adapted from the software Deformetrica where we introduce constraints to remain control points pooled during optimization. An example of result is shown on Figure 7.

\section{Conclusion}

We constructed a mathematical framework for generic deformation modules, which is stable under combination. Large deformations can be built then by integrating vector fields generated by these modules. By defining a cost on module we allow optimal deformations to come from geodesic paths in a sub-Riemannian manifold. We presented several examples of modules and geodesics. The construction allows an easy interpretation of the computed deformation and the incorporation of anatomical prior, so that this work may have important applications for the analysis of biological shapes.

\section{References}

1. A. Agrachev, U. Boscain, G. Charlot, R. Ghezzi, and M. Sigalotti. Two-dimensional almost-riemannian structures with tangency points. In Decision and Control, 2009 held jointly with the 2009 28th Chinese Control Conference. CDC/CCC 2009. Proceedings of the 48th IEEE Conference on, pages 4340-4345. IEEE, 2009.

2. S. Arguillere. Géométrie sous-riemannienne en dimension infinie et applications à l'analyse mathématique des formes. PhD thesis, Paris 6, 2014.

3. V. Arsigny, O. Commowick, N. Ayache, and X. Pennec. A fast and log-euclidean polyaffine framework for locally linear registration. Journal of Mathematical Imaging and Vision, 33(2):222-238, 2009.

4. J. Ashburner. A fast diffeomorphic image registration algorithm. Neuroimage, 38(1):95-113, 2007. 
5. N. Charon and A. Trouvé. The varifold representation of non-oriented shapes for diffeomorphic registration. arXiv preprint arXiv:1304.6108, 2013.

6. S. Durrleman, M. Prastawa, G. Gerig, and S. Joshi. Optimal data-driven sparse parameterization of diffeomorphisms for population analysis. In Information Processing in Medical Imaging, pages 123-134. Springer, 2011.

7. U. Grenander. Elements of pattern theory. JHU Press, 1996.

8. H. Jacobs. Symmetries in lddmm with higher order momentum distributions. arXiv preprint arXiv:1306.3309, 2013.

9. S. Joshi, P. Lorenzen, G. Gerig, and E. Bullitt. Structural and radiometric asymmetry in brain images. Medical Image Analysis, 7(2):155-170, 2003.

10. M. I. Miller, A. Trouvé, and L. Younes. On the metrics and euler-lagrange equations of computational anatomy. Annual review of biomedical engineering, 4(1):375-405, 2002.

11. M. I. Miller, L. Younes, and A. Trouvé. Diffeomorphometry and geodesic positioning systems for human anatomy. Technology, 2(01):36-43, 2014.

12. L. Risser, F. Vialard, R. Wolz, M. Murgasova, D. D. Holm, and D. Rueckert. Simultaneous multi-scale registration using large deformation diffeomorphic metric mapping. Medical Imaging, IEEE Transactions on, 30(10):1746-1759, 2011.

13. D. Rueckert, P. Aljabar, R. A. Heckemann, J. V. Hajnal, and A. Hammers. Diffeomorphic registration using b-splines. In Medical Image Computing and ComputerAssisted Intervention-MICCAI 2006, pages 702-709. Springer, 2006.

14. C. Seiler, X. Pennec, and M. Reyes. Capturing the multiscale anatomical shape variability with polyaffine transformation trees. Medical image analysis, 16(7):1371-1384, 2012.

15. S. Sommer, F. Lauze, M. Nielsen, and X. Pennec. Sparse multi-scale diffeomorphic registration: the kernel bundle framework. Journal of mathematical imaging and vision, 46(3):292-308, 2013.

16. S. Sommer, M. Nielsen, S. Darkner, and X. Pennec. Higher-order momentum distributions and locally affine lddmm registration. SIAM Journal on Imaging Sciences, 6(1):341-367, 2013.

17. M. Vaillant, M. I. Miller, L. Younes, and A. Trouvé. Statistics on diffeomorphisms via tangent space representations. NeuroImage, 23:S161-S169, 2004.

18. T. Vercauteren, X. Pennec, A. Perchant, and N. Ayache. Symmetric Log-Domain Diffeomorphic Registration: A Demons-based Approach. In D. Metaxas, L. Axel, G. Fichtinger, and G. Székely, editors, Medical Image Computing and Computer Assisted Intervention, volume 5241, pages 754-761, New York, United States, Sept. 2008. Springer. pmid 18979814.

19. L. Younes. Constrained diffeomorphic shape evolution. Foundations of Computational Mathematics, 12(3):295-325, 2012.

20. W. Zhang, J. A. Noble, and J. M. Brady. Adaptive non-rigid registration of real time 3d ultrasound to cardiovascular $\mathrm{mr}$ images. In Information Processing in Medical Imaging, pages 50-61. Springer, 2007. 